\title{
Carbon-derived Substrate Accelerated the Biodegradation of Low-density Polyethylene
}

\author{
Crystal Radinski \\ Webber Academy, 151593 St SW, Calgary, AB T3H 4A8, Canada; cradinski@hotmail.com
}

\begin{abstract}
Plastic is a commonly used carbon-based product that poses a major environmental pollution hazard due to its poor biodegradability. The natural environment is rich in organic carbon sources. Only few microorganisms have developed abilities to disintegrate heavy plastic molecules, and their lytic capabilities are generally poor. Microorganisms select carbon molecules that require less energy to digest. As bacteria are highly adaptable organisms, it is hypothesized that bacterial plastic biodegradation activity could be stimulated by limiting carbon sources to plastic and forcing bacteria to upregulate necessary metabolic pathways. Bacteria with natural abilities to decompose plastic from three different ecosystems in Alberta, Canada (forest, river, and farm) were cultured in an artificially created environment with limited carbon access. Pseudomonas sp. demonstrated the ability to accelerate LDPE biodegradation from $1.39 \%$ in carbon-saturated to $21.335 \%$ in carbon-restricted mediums in three months. The results of our study suggest that plastic pollution could be reduced by increasing its biodegradation in dedicated composters. Finding a solution to the overwhelming waste issue is critical to the planet's health.

KEYWORDS: Microbiology; Bacteriology; Biodegradation; Pseudomonas; Plastic.
\end{abstract}

\section{Introduction}

Plastic is a carbon-based polymer of high molecular mass, usually produced from petroleum. It is a very versatile modern material, widely used in the manufacturing of a large range of products from automobiles and buildings to toys and cosmetics. ${ }^{1}$ Plastic poses a significant environmental pollution threat due to its resistance to biodegradation. In 2009, it was estimated that $10 \%$ of modern waste was in the form of plastics. ${ }^{2}$ In marine areas, plastic debris reaches $50 \%-80 \%$ of total waste. ${ }^{3}$ Indeed, a study done in 2014 estimated that 245,000 tons of plastic waste were floating in the ocean. ${ }^{4}$ Plastic pollution has been described as the "single greatest threat" to marine animals and birds. ${ }^{5}$ Moreover, animals of different sizes are affected by plastic in a variety of ways. When consumed, plastic blocks digestion in animal's stomachs and can cause starvation. Large marine mammals can be killed by entanglement. Poisoned animals can harm human food supplies as well. Additionally, some additives may be cancerous to humans. ${ }^{5}$ Additives such as phthalates and BPA can provoke hormonal dysregulations like thyroid, fertility, and skin disorders. ${ }^{5}$

The disposal of plastics is extremely difficult. Despite being a carbon-based material, plastics are synthetically produced and, because of the high molecular weight of their hydrophobic long carbon chains, are not easily breakable by microbial enzymes. Several bacteria, yeast, and fungi have been identified to have a weak ability to breakdown plastic. ${ }^{6}$ Research has been done to create biodegradable plastics, but the resulting products are expensive. ${ }^{7}$ In a landfill, plastic becomes a carbon sink, and incineration increases carbon emissions. ${ }^{8}$ If the plastic is incinerated, it also increases carbon emissions and produces polychlorinated di benzo-p-dioxins, a carcinogen. ${ }^{9}$ Recycling is difficult and labor intensive. During 2008 in the US, only $6.5 \%$ of plastic waste were recycled, while $85.5 \%$ were discard- ed in landfills. ${ }^{10}$ Because of plastic's intractable resistance to biodegradation, the disposal strategies are crucial and demand attention.

Low-density polyethylene (LDPE) is one of the most used plastics. ${ }^{1}$ LDPE biodegradation, the safest way to treat waste, is limited by microbial abilities to break strong polymer chains. Several bacteria have demonstrated a natural ability to biodegrade plastics. Ideonella sakaiensis 201-F6 is able to use polyethylene terephthalate (PET) as its major energy and carbon source. ${ }^{14}$ Bacillus brevis showed ability to degrade polycaprolactone. ${ }^{16}$ Streptomyces can degrade PHB, poly(3-hydoxybutyarate-co-3-hydroxyvalerate), and polyester.

This degradation occurs in sequential steps. Firstly, bio-deterioration alters the chemical and physical properties of the polymer before bio-fragmentation can use enzymatic cleavage to breakdown polymers to a simpler form. Then, in the assimilation phase, microorganism's uptake polymer molecules produce oxidized metabolites $\left(\mathrm{CO}_{2}, \mathrm{CH}_{4}, \mathrm{H}_{2} \mathrm{O}\right)$. In the final step of degradation, mineralization occurs. ${ }^{18}$

Several natural metabolic pathways leading to plastic degradation were identified. Ideonella sakaiensis produces two enzymes, terephthalic acid and ethylene glycol allow it to efficiently convert PET into environmentally friendly monomers. ${ }^{17}$ Rhodococcus ruber uses copper-binding laccase in enzymatic degradation of polythene. ${ }^{19}$

However, the activity of enzymes participating in the synthetic polymer's fermentation has been shown to be weak. This could be possibly explained by the availability of other biopolymers, such as cellulose, requiring less energy to digest. ${ }^{11}$ Nevertheless, since natural pathways for plastic fermentation exist, it might be possible to stimulate their activity to increase plastic natural and safe decay. It is possible that re 
stricting bacterial sources of carbon to plastic will cause the microorganisms to adapt and naturally activate their plastic biodegrading ability. Providing synthetic polymers, like plastic, as a sole carbon source will stimulate bacteria to adapt to these carbon sources, forcing the upregulation of plastic-degrading enzymes to allow the metabolism of synthetic polymers and, therefore, help decompose plastic into environmentally neutral monomers faster.

\section{Methods}

In the first stage of this study, to obtain bacteria with natural plastic biodegradation ability, soils from three sources were utilized: an Alberta cattle farm, Elbow riverbed, and an Alberta mixed forest. It was previously demonstrated that biodegradation begins with the colonization of microorganisms on the surface of the material. ${ }^{12}$ Soil microorganisms were cultured in nature-like landfill conditions, recreated in a miniature, enclosed ecosystem. Winogradsky columns containing the soil, rainwater, and $30 \mathrm{~cm}^{2}$ LDPE strips were used. Plastic samples were incubated for three months in a room with indirect natural light and allowed temperature fluctuation consistent with the Alberta climate. This allowed the bacterial strains capable of using LDPE as a carbon source to colonize the plastic. After three months, biofilm was observed to form on the surfaces of the plastic. Three colonies formed on the plastic incubated in the forest ecosystem, three in the river and six in the farm Winogradsky columns. Bacterial samples were collected and cultured on agar in petri dishes to isolate monoclonal bacterial cultures with the presumed natural plastic-degradation ability. Twelve colonies were isolated and used as the study subjects in the second stage of the study.

In the second stage, to assess each isolate's plastic biodegradation activity (BA), two test groups were created with carbon-restricted and carbon-enriched media. Each bacterial culture was with an LDPE sample in two mediums for three months. In three months, the BA in the two groups was compared. Biodegradation is characterized by signs of disintegration such as a loss of weight, change in physical properties, carbon dioxide production etc. In this study, BA was monitored as a percentage of plastic weight loss after three months of incubation. BA in the control group served as an indicator of baseline biodegradation activity for a given bacterial strain.

\section{Procedure:c}

Twenty-five $15 \mathrm{ml}$ test tubes were sterilized and filled with $10 \mathrm{ml}$ sterile Bushnell-Haas medium $\left(\mathrm{MgSO}_{4}-0.2 \mathrm{~g}\right.$, $\mathrm{CaCl}_{2}-0.02 \mathrm{~g}, \mathrm{KH}_{2} \mathrm{PO}_{4}-1 \mathrm{~g}, \mathrm{FeCl}_{2}-0.05 \mathrm{~g}, \mathrm{NH}_{4} \mathrm{NO}_{3}-1 \mathrm{~g}$, distilled water - $1000 \mathrm{ml}, \mathrm{pH}-7.0$ ) to provide necessary elements for bacterial growth other than carbon. Twentyfive strips of LDPE plastic $(1 \times 3 \mathrm{~cm})$ were prepared: washed with distilled water, sterilized with $70 \%$ ethanol for 5 minutes and subsequently dried in an incubator at $25^{\circ} \mathrm{C}$ for 24 hours. Plastic samples were then weighed with a Mettler Toledo model AL204 analytical balance and added to each test tube. In the carbon-restricted group, twelve monoclonal cultures were incubated in a medium where plastic was the only source of carbon. In the carbon-saturated test group, starch was added to the medium as a source of easy-to-digest carbon to simulate a carbon-rich natural environment (soil). A colony representative of each isolate was inoculated into two test tubes (one in carbon-restricted and one in carbon-enriched groups). Three samples of bacteria were collected from the forest soil, three from the river soil and six from the farm soil agar dishes. A plastic strip was also added to one test tube with a carbon-deprived medium in the absence of inoculum to account for any abiotic losses. Tubes were labelled, and medium and soil type data was recorded in a corresponding logbook. Tubes were incubated for three months at $25^{\circ} \mathrm{C}$. The inoculation and incubation were carried out under aseptic conditions. After three months, incubated plastic samples were removed and cleaned of the colonizing bacteria and their metabolism byproducts with water and 70\% ethanol. Samples were air-dried for 24 hours before the weight was recorded.

Biodegradation activity, as a percent of LDPE weight loss, was measured for each test sample and compared between respective cultures in carbon-saturated and carbon-restricted mediums. Cultures (isolates) with the most accelerated biodegradation activities were identified following sample preparation using the polymerase chain reaction $(\mathrm{PCR})$ of the $16 \mathrm{~S}$ rRNA gene and subsequent DNA sequencing at the University of Calgary.

\section{- Results and Discussion}

In the first stage of the study, LDPE samples with bacteria from three Alberta natural environments were colonized to isolate bacteria with presumed natural biodegrading abilities. Twelve bacterial cultures were isolated from colonies formed on LDPE.

A summarized weight loss of the plastic samples after three months of incubation in three natural soils was created to provide a better understanding of the rate of natural biodegradation (Table 1). In the first stage, the most active BA was detected in the soil collected from Alberta mixed forest at $6.01 \%$, compared to farm soil at $3.13 \%$ and from river at $1.27 \%$.

Table 1: Weight (in grams) of plastic samples.

\begin{tabular}{|l|c|c|c|c|c|}
\hline $\begin{array}{l}\text { Soil } \\
\text { source }\end{array}$ & initial weight $(\mathrm{g})$ & weight in 3 months $(\mathrm{g})$ & weight loss $(\mathrm{g})$ & $\begin{array}{l}\text { \% weight at 3 } \\
\text { months }\end{array}$ & BA \% \\
\hline Forest & 0.2793 & 0.2625 & 0.0168 & 93.984 & 6.015 \\
\hline Farm & 0.2617 & 0.2535 & 0.0082 & 96.866 & 3.133 \\
\hline Riverbed & 0.2198 & 0.217 & 0.0028 & 98.726 & 1.273 \\
\hline
\end{tabular}

In the second stage of the experiment, LDPE samples were submerged into carbon-deprived substrate and separately exposed to twelve bacterial isolates with presumed plastic degenerative abilities cultured in the first stage of the study. The results for the carbon-deprived group are summarised in Table 2 and for the carbon-enriched group in Table 3. In three months, all twelve cultures demonstrated an increase in biodegradation activity. A plastic strip incubated in the medium with no inoculum was compared to account for any abiotic losses that did not show any weight loss.

To determine statistical significance in the groups'BA differences, a student t-test was performed.

Twelve bacterial cultures demonstrated statistically significantly increased biodegradation activity in the carbon-deprived 
group showed a statistically significant acceleration of biodegrading abilities of microbial cultures.

$\mathrm{t}$-value $=-2.92202$

$\mathrm{p}$-value $=.003948$

Table 2: Plastic weight loss in response to the exposure to monoclonal cultures in carbon-deprived medium.

\begin{tabular}{|r|l|r|r|r|r|r|}
\hline \multicolumn{1}{|l|}{ culture\# } & source & initial wt./g & wt. at 3 mo./g & wt. loss $/ \mathrm{g}$ & $\begin{array}{l}\text { Wt. at 3 mo. } \\
(\%)\end{array}$ & BA (\%) \\
\hline 1 & forest & 0.0989 & 0.0778 & 0.0211 & 78.6653 & 21.3346 \\
\hline 2 & farm & 0.0669 & 0.0663 & 0.0006 & 99.1031 & 0.8968 \\
\hline 3 & farm & 0.0675 & 0.0643 & 0.0032 & 95.2592 & 4.7407 \\
\hline 4 & farm & 0.0625 & 0.0609 & 0.0016 & 97.44 & 2.56 \\
\hline 5 & farm & 0.0535 & 0.0521 & 0.0001 & 97.3831 & 2.6168 \\
\hline 6 & riverbed & 0.0575 & 0.0574 & 0.0001 & 99.826 & 0.1739 \\
\hline 7 & forest & 0.0603 & 0.0519 & 0.0084 & 86.0696 & 13.9303 \\
\hline 8 & forest & 0.0503 & 0.0449 & 0.0054 & 89.2644 & 10.7355 \\
\hline 9 & riverbed & 0.0517 & 0.0506 & 0.0011 & 97.8723 & 2.1276 \\
\hline 10 & riverbed & 0.0701 & 0.0685 & 0.0016 & 97.7175 & 2.2824 \\
\hline 11 & farm & 0.0679 & 0.0624 & 0.0055 & 91.8998 & 8.1001 \\
\hline 12 & farm & 0.0636 & 0.0605 & 0.0031 & 95.1257 & 4.8742 \\
\hline
\end{tabular}

Table 3: Plastic weight loss in monoclonal culture in carbon-saturated medium (control).

\begin{tabular}{|c|c|c|c|c|c|c|}
\hline culture\# & source & initial wt. $/ \mathrm{g}$ & wt. at $3 \mathrm{mo} . / \mathrm{g}$ & wt. loss $/ g$ & $\begin{array}{l}\text { Wt. at } 3 \text { mo. } \\
(\%)\end{array}$ & $\mathrm{BA}(\%)$ \\
\hline 1 & forest & 0.0789 & 0.0778 & 0.0011 & 98.60583 & 1.3941 \\
\hline 2 & farm & 0.0769 & 0.0763 & 0.0006 & 99.2197 & 0.7802 \\
\hline 3 & farm & 0.0647 & 0.0643 & 0.0032 & 99.3817 & 0.6182 \\
\hline 4 & farm & 0.0602 & 0.0599 & 0.0003 & 99.5016 & 0.4983 \\
\hline 5 & farm & 0.0759 & 0.0755 & 0.0004 & 99.4729 & 0.527 \\
\hline 6 & riverbed & 0.0575 & 0.057 & 0.0005 & 99.1304 & 0.8695 \\
\hline 7 & forest & 0.0601 & 0.0594 & 0.0007 & 98.8352 & 1.1647 \\
\hline 8 & forest & 0.0872 & 0.0861 & 0.0011 & 98.7385 & 1.2614 \\
\hline 9 & riverbed & 0.0517 & 0.0512 & 0.0005 & 99.0328 & 0.9671 \\
\hline 10 & riverbed & 0.0701 & 0.0698 & 0.0003 & 99.572 & 0.4279 \\
\hline 11 & farm & 0.0679 & 0.0671 & 0.0008 & 98.8217 & 1.1782 \\
\hline 12 & farm & 0.0636 & 0.0632 & 0.0004 & 99.371 & 0.6289 \\
\hline
\end{tabular}

The result of the study supported the hypothesis that providing plastic as the sole source of carbon stimulation or providing a way for mutating bacteria's natural metabolic pathways, allows digestion of synthetic polymers and acceleration of LDPE plastic biodegradation. The study showed a promising microorganisms' ability to decay LDPE at a faster rate than currently occur in nature.

\section{Culture identification:}

Four cultures with the greatest change in BA to be identified with PCR and DNA sequencing were selected. Three cultures isolated from forest originated sample $(\# 1, \# 7, \# 8)$ showed accelerated activity at $21.33 \%, 13.93 \%$ and $10.73 \%$ compared to control bioactivity of respective cultures $1.39 \%, 1.16 \%$ and $1.26 \%$. Fourth active sample collected from farmland (\#11) demonstrated bioactivity of $8.1 \%$ compared to the control bioactivity of $1.17 \%$.

A multitude of Pseudomonas species were identified in culture\#1. Comamonas, Rhizobium, and Agrobacterium were identified in cultures \# 7, \#8 and \#11 (Table 4).

Table 4: Top four cultures with the highest BA acceleration.

\begin{tabular}{|l|l|l|l|}
\hline Soil source & \multicolumn{1}{|c|}{ Microbial strain } & \multicolumn{1}{|c|}{$\begin{array}{c}\text { BA in carbon-deprived } \\
\text { medium }\end{array}$} & \multicolumn{1}{|c|}{$\begin{array}{c}\text { BA in carbon-saturated } \\
\text { medium }\end{array}$} \\
\hline forest & Pseudomonas & $21.34 \%$ & $1.39 \%$ \\
\hline forest & Comamonas & $13.93 \%$ & $1.16 \%$ \\
\hline forest & Rhizobium & $10.73 \%$ & $1.26 \%$ \\
\hline farm & Agrobacterium & $8.1 \%$ & $1.17 \%$ \\
\hline
\end{tabular}

All identified organisms are known soil organisms, some with pollutant-degrading metabolism (Pseudomonas and Comamonas). Pseudomonas have very simple nutritional require ments. One of the most striking properties of Pseudomonas species is the ability to use a wide variety of organic carbon and energy sources. Some Pseudomonas species utilize over 100 different compounds, including many simple sugars, fatty acids, alcohols, glycolic, amino acids, and many other compounds not fitting in any other categories. ${ }^{14}$ Pseudomonas contain multiple different enzymes and capable of using multiple metabolic pathways to allow different types of carbon digestion. Pseudomonas are known for a weak lytic activity necessary to break down long polymers' chains. For example, Pseudomonas can degrade polythene, PVC, PHB, poly(3-hydroxybutyrate-co-3-mercaptopropionate), and poly(3-droxypropionate). Since Pseudomonas species possess such a wide variety of metabolic mechanisms, in order to survive, the bacteria can be highly adaptive and activate their lytic activity.

\section{Study limitations:}

Identification and isolation of bacteria with a natural ability to decompose plastic was a challenging process. There were billions of bacteria and millions of cultures in the original soil samples. The selection process was based on the assumption that bacteria would adhere/colonize plastic. Samples were collected from the plastic surface. However, it is possible that some active cultures were not captured. It does not change the outcome of this study as the focus was on a variety of bacteria with a different degree of enzyme activity. The focus of the study was to research the ability of bacteria to adapt in a carbon-restricted environment. Therefore, it is possible that even more active in lytic activity bacteria could exist in a natural environment.

Due to limited resources, it was not possible to replicate each test condition. In the future, a goal for this project would be to repeat the test with the study's most accelerated culture Pseudomonas sp. Creating three to five replicates would strengthen the statistical analysis of the study. Also, limited resources precluded the use of PCR to identify all 12 isolates. It would be interesting to know if other tests contained the same species but did not accelerate at the same rate.

\section{- Conclusion}

Some bacteria's natural metabolic pathways could be stimulated to allow the digestion of synthetic polymers and accelerated biodegradation of LDPE plastic. The current LDPE recycle rate is very low (less than 10\%) due to the labor-intensive recycling technique. ${ }^{13}$ The study showed a promising ability of the microorganisms to decay LDPE at a faster rate than it currently occurs in nature. To achieve that, synthetic polymers need to be treated as selective compost to stimulate the microorganism's lytic activity. A designated site for proper disposal of rejected recyclables could help reduce the planet's overall pollution.

\section{- Acknowledgements}

I'd like to thank my mentor Dr. Lisa Geig and my parents for their endless support and encouragement.

\section{References}

1. Market Study: Polyethylene LDPE, 3rd edition; Ceresana, 2020. 2. Thompson RC, Swan SH, Moore CJ, vom Saal FS. Our Plastic Age. Philos. Trans. R. Soc. Lond., B, Biol. Sci 2009. 364 (1526): 1973-6. 
3. Barnes DK; Galgani F; Thompson RC, Barlaz M. Accumulation and fragmentation of plastic debris in global environments. Philos. Trans. R. Soc. Lond., B, Biol. Sci. 2009, 364 (1526): 1985-98.

4. Laura Parker. Eight Million Tons of Plastic Dumped in Ocean Every Year. National Geographic. 2015.

5. Emily J. North, Rolf U. Halden. Plastics and Environmental Health: The Road Ahead. Rev Environ Health. 2013; 28(1): 1-8.

6. Cacciari I; Quatrini P; Zirletta G; Mincione E; Vinciguerra V; Lupattelli P; Giovannozzi Sermanni G. Isotactic polypropylene biodegradation by a microbial community: physicochemical characterization of metabolites produced. Applied and Environmental Microbiology. 1993.

7. Vink, E. T. H.; Rabago, K. R.; Glassner, D. A.; Gruber, P. R. Polymer Degradation and Stability. 2003, 80, 403-419.

8. Halden,R. Plastics and Health Risks. Annual Review of Public Health. 2010; 31:179-94.

9. Burd D. Plastic Not Fantastic. 2008 http://wwsef.uwaterloo.ca/ archives/2008/08BurdReport.pdf

10.Margaret Weigel. Energy and Economic Value of Non-recycled Plastics and Municipal Solid Wastes. The Journalist's

Resource. 2017.

11.M Madigan, J Martinko. Brock Biology of Microorganisms, 11th edition. International Microbiology. 2005.

12.Roy PK, Surekha P, Tulsi E, Deshmukh C, Rajagopal C. Degradation of abiotically aged LDPE films containing prooxidant by bacterial consortium. Polym Degrad Stab. 2008; 93:1917-1922.

13.Margaret Weigel. Energy and Economic Value of Nonrecycled Plastics and Municipal Solid Wastes. The Journalist's Resource. 2011.

14. Shosuke Yoshida et al. A bacterium that degrades and assimilates poly (ethylene terephthalate). Science 11.2016: 6278,1196-1199

15.Maria Jose Cardenas Espinosa et al. Toward Biorecycling: Isolation of a Soil Bacterium That Grows on a Polyurethane Oligomer and Monomer. Pub Med. 2020

16.Hadad D, Geresh S, Sivan A .Biodegradation of polyethylene by the thermophilic bacterium Brevibacillus borstelensis. J Appl Microbiol. 2005, 98(5):1093-1100

17.Lee at al. Biodegradation of degradable plastic polyethylene by Phanerochaete and Streptomyces species. Appl Environ Microbiol. 1991, 57(3):678-85.

18.Singh B, Sharma N. Mechanistic implications of plastic degradation. Polym Degrad Stab. 2008. 93:561- 584

19.Santo M, Weitsman R, Sivan A. The role of the copper-binding enzyme- laccase- in the biodegrading of polyethylene by the actinomycete Rhoducoccus ruber. Int Biodeterior Biodegrad 2013. 84:204-210.

\section{- Author}

Crystal Radinski began her scientific problem-solving experimentations at the age of twelve and presented her successful work at several international competitions. Crystal enjoys equestrian show jumping, traveling, and spending time with friends. She hopes to make a positive difference in our society by pursuing her interests in mental health and psychology. 